\title{
A nanomedicine approach enables co-delivery of cyclosporin A and gefitinib to potentiate the therapeutic efficacy in drug-resistant lung cancer
}

\author{
Weidong Han ${ }^{1}$, Linlin Shi ${ }^{1}$, Lulu Ren ${ }^{1}$, Liqian Zhou ${ }^{2}$, Tongyu $\mathrm{Li}^{2}$, Yiting Qiao ${ }^{2}$ and Hangxiang Wang ${ }^{2}$
}

\begin{abstract}
Drug resistance, accounting for therapeutic failure in the clinic, remains a major challenge to effectively manage cancer. Cyclosporin A (CsA) can reverse multidrug resistance (MDR), especially resistance to epidermal growth factor receptor tyrosine kinase inhibitors. However, the application of both drugs in cancer therapies is hampered by their poor aqueous solubility and low bioavailability due to oral administration. CsA augments the potency of gefitinib (Gef) in both Gef-sensitive and Gef-resistant cell lines. Here, we show that the simultaneous encapsulation of CsA and Gef within polyethylene glycol-block-poly ( $D$, L-lactic acid) (PEG-PLA) produced a stable and systemically injectable nanomedicine, which exhibited a sub-50-nm diameter and spherical structures. Impressively, the co-delivery of therapeutics via single nanoparticles (NPs) outperformed the oral administration of the free drug combination at suppressing tumor growth. Furthermore, in vivo results indicated that CsA formulated in NPs sensitized Gef-resistant cells and Gefresistant tumors to Gef treatment by inactivating the STAT3/Bcl-2 signaling pathway. Collectively, our nanomedicine approach not only provides an alternative administration route for the drugs of choice but also effectively reverses MDR, facilitating the development of effective therapeutic modalities for cancer.
\end{abstract}

Signal Transduction and Targeted Therapy (2018)3:16; https://doi.org/10.1038/s41392-018-0019-4

\section{INTRODUCTION}

Despite significant progress in treating cancer, drug resistance remains a major challenge to effectively manage this disastrous disease. Drug resistance also accounts for the therapeutic failure and relapse in over $90 \%$ of cancer patients. ${ }^{1,2}$ Cancer drug resistance can be intrinsic or acquired as a response to repeated chemotherapy. Tremendous efforts have been directed toward overcoming this obstacle, including the following strategies: (i) down-regulating the activity of drug efflux pumps with specific inhibitors $^{3-5}$ or RNA interference technology; ${ }^{6-10}$ (ii) developing new therapeutics that are less susceptible to drug resistance; ${ }^{11,12}$ and (iii) combining two or more agents into one treatment scheme, one of which usually rewires specific cell signaling pathways to sensitize the cells and subsequently improves the therapeutic efficacy of the other drugs. ${ }^{13-16}$ The latter antiresistance approach is particularly attractive because numerous studies have shown that a single magic bullet strategy is a rare commodity in treating cancer. Therefore, drug combinations offer the opportunity to target different pathways in specific populations of cancer cells, thereby maximizing the therapeutic efficacy or overcoming drug resistance mechanisms. However, it is extremely difficult to reconcile the pharmacokinetics, bio-distribution, and cellular uptake of individual drugs that possess distinct physiochemical properties. Therefore, current clinical combinatorial therapy, i.e., simply combining different free drugs, is far from a perfect regimen for cancer patients.
To address these therapeutic challenges, packaging multiple drugs or drug candidates into a single nanocarrier is of interest to achieve synergistic activity. In addition, due to the leaky tumor microvasculature coupled with impaired intratumoral lymphatic drainage, a unique characteristic known as the enhanced permeability and retention (EPR) effect can be leveraged to facilitate the preferential accumulation of nanotherapeutics at tumor site(s), barring drug distribution in normal tissues. ${ }^{17-21}$ Thus, numerous nanostructured vehicles have been explored to efficiently and safely deliver anticancer therapeutics. ${ }^{22-25}$ Particularly, polymeric nanoparticles, as nanocarriers derived from a broad range of amphiphilic copolymers, have shown great potential for drug delivery. ${ }^{26-29}$ Traditionally, these polymeric matrices exhibit excellent biocompatibility and biodegradability, thus avoiding long-term biosafety concerns. ${ }^{30}$ More importantly, multiple structurally diverse hydrophobic drugs can be simultaneously encapsulated within a single platform. Exploiting these nanomedicines has been successful in many instances. ${ }^{25,31}$ However, only a few studies have been conducted to elucidate the effect of delivering a chemosensitizer and a molecular targeted agent (MTA) via a single injectable nanomedicine on therapeutic outcomes in cancer.

Activation of the transcription factor signal transducer and activator of transcription 3 (STAT3) in response to diverse stimuli can promote tumor cell survival and enhance tumor stem cell-like properties. $^{32-34}$ Thus, the targeted disruption of this signal

\footnotetext{
${ }^{1}$ Department of Medical Oncology, Sir Run Run Shaw Hospital, School of Medicine, Zhejiang University, 310016 Hangzhou, China and ${ }^{2}$ The First Affiliated Hospital, Collaborative

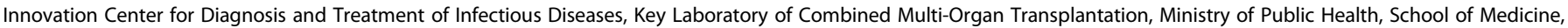
Zhejiang University, 310003 Hangzhou, China

Correspondence: Hangxiang Wang (wanghx@zju.edu.cn)
}

Received: 13 February 2018 Revised: 26 April 2018 Accepted: 15 May 2018

Published online: 22 June 2018 
transduction pathway has emerged as a potential therapeutic strategy for cancer. Our previous study has suggested that STAT3 is also associated with chemoresistance against Gef, an epidermal growth factor receptor tyrosine kinase inhibitor (EGFR-TKI), in lung cancer cells. ${ }^{35}$ Strikingly, drug resistance can be partially abolished by the addition of CsA, an Food and Drug Administration (FDA)approved drug to prevent immune rejection after organ transplantation, ${ }^{36}$ via STAT3 pathway inactivation. We hypothesized and demonstrated that the individual administration of these two drugs could result in reduced drug synergy and, thus, reduce the therapeutic benefits due to uncontrolled pharmacokinetic profiles. However, beyond this preliminary demonstration, we did not study the combinatorial therapeutic effects or attempt the co-encapsulation of these drugs for systemic injection. Therefore, we envisioned that nanomedicine approaches could significantly improve the pharmacokinetics and precisely tailor the intracellular interplay of two drugs, thereby potentiating synergistic efficacy compared with the oral administration or either drug alone.

Herein, by exploiting the intrinsic hydrophobicity of drug payloads, we simultaneously encapsulated a chemosensitizer (i.e., CsA) ${ }^{37}$ and a MTA into polymeric NPs comprising PEG-PLA clinically approved by the FDA. ${ }^{38-40}$ The resulting NPs exhibited significantly improved drug solubility and stability, thereby enabling the systemic injection of both drugs within a single formulation. Moreover, we thoroughly examined the role of CsA in reversing Gef resistance both in vitro and in vivo. In addition to providing an alternative administration route for the drugs of choice, our study also demonstrated that systematically injectable nanomedicines could be utilized as practical and effective therapeutic modalities in oncology.

\section{RESULTS}

Preparation and characterization of an amphiphilic copolymerbased nanocarrier encapsulating one or two drugs

Both chemotherapeutics (CsA and Gef) are highly water insoluble. The solubility of CsA and Gef in deionized (DI) water is less than $0.1 \mathrm{mg} / \mathrm{mL}{ }^{41,42}$ The large void space of the inner core of PEG-PLA NPs was expected to accommodate the integration of the two drugs, thus enabling their systemic injection. To test this rationale, we used a nanoprecipitation procedure to entrap one or two drugs using $\mathrm{PEG}_{5 \mathrm{k}}-\mathrm{PLA}_{8 \mathrm{k}}$ matrices (Fig. 1a). As expected, blending the $\mathrm{PEG}_{5 \mathrm{k}}-\mathrm{PLA}_{8 \mathrm{k}}$ copolymer with CsA, Gef or both CsA and Gef produced transparent solutions rather than precipitates, suggesting the successful encapsulation of chemically dissimilar therapeutics in PEG-PLA NPs. TEM-based morphology studies revealed the formation of spherical nanostructures with a diameter of approximately $25 \mathrm{~nm}$ (Fig. 1b). These results were further validated by dynamic light scattering (DLS). DLS analysis showed that the CsA loaded NPs (CsA-NPs), Gef-loaded NPs (Gef-NPs) and CsA- and Gef-coloaded NPs (CsA/ Gef-NPs) had average hydrodynamic diameter $\left(D_{\mathrm{H}}\right)$ values of $25.7 \pm 6.3,42.4 \pm 14.9$, and $37.1 \pm 13.1 \mathrm{~nm}$, respectively (Fig. 1c, d). More importantly, CsA/Gef-NPs exhibited remarkable colloidal stability in phosphate-buffered saline (PBS) or in the presence of $20 \%$ fetal bovine serum (FBS) over long-term incubation (at least 1 week) when stored at room temperature (Fig. S1). Only negligible variations in $D_{\mathrm{H}}$ were observed for CsA/Gef-NPs as confirmed by DLS analysis.

In vitro cytotoxicity and chemosensitizing effects of nanomedicine Previous studies have indicated that CsA can enhance the potency of MTAs, including Gef. ${ }^{35,43-45}$ We also demonstrated the effect of CsA on sensitizing non-small cell lung cancer (NSCLC) cells to free Gef in Fig. S2. However, we are the first to attempt to verify whether the combination of $\mathrm{CsA}$ and Gef could produce a synergistic effect against cancer cells, especially Gef-resistant
NSCLC cells, when formulated into NPs and co-delivered. To assess this effect, three NSCLC cell types, including EGFR-TKI-sensitive PC9, acquired-resistance PC-9 and primary-resistance H1975 cells, were investigated in vitro. After treatment with Gef in the presence or absence of CsA via NPs, cell viability was confirmed by the MTT assay. As shown in Fig. 2a, a remarkable synergistic effect was observed in PC-9 cells even at a low NP concentration of CsA ( $1 \mu \mathrm{M}$ CsA equivalent concentration), whereas CsA-NPs alone did not exhibit obvious cytotoxicity. Specifically, the addition of CsA (e.g., $1 \mu \mathrm{M}$ ) to the NP formulation reduced the half-maximal inhibitory concentration $\left(\mathrm{IC}_{50}\right)$ values of Gef from 29.3 to $19.3 \mu \mathrm{M}$ and from 18.5 to $9.3 \mu \mathrm{M}$ in Gef acquired resistant PC-9 (PC-9-GR) and primarily Gef resistant $\mathrm{H} 1975$ cells, respectively (Fig. 2b, c). The cytotoxic effect of the drug-loaded NPs was comparable or even superior to that of the combination of both free drugs in all the tested NSCLC cell types (Fig. 2d-f), indicating that the nanoformulation of the two drugs did not impair the synergistic antitumor effect in vitro.

To further investigate whether the enhanced sensitization to Gef by CsA when co-delivered by NPs was a consequence of increased apoptosis, Gef-NPs-treated cells were double stained with propidium iodide (PI)/Annexin V-FITC and quantified by flow cytometry. Treatment with Gef-NPs induced apoptosis, resulting in apoptotic ratios of 20.06, 5.27 and $26.98 \%$ in PC-9, PC-9-GR and H1975 cells, respectively. However, the addition of CsA to the NP formulation substantially enhanced the apoptotic ratios to 41.65 , 16.41 , and $37.32 \%$ in the above three cell lines, respectively (Fig. 3a-f). The effect of free CsA on sensitizing cells to free Gef was also confirmed in NSCLC cells (Fig. S3). The synergistic cytotoxic effect of the nanomedicines was further detected by LIVE/DEAD staining. As summarized in Fig. 3g-l, treatment with CsA/Gef-NPs led to 1.8- to 2.3-fold more cell death than treatment with Gef-NPs in all NSCLC cells.

Additionally, the EdU incorporation assay was applied to examine the effect of the nanomedicine co-encapsulating both drugs on cancer cell proliferation compared with that of the single-drug NP formulations. As presented in Fig. 4a-f, treatment with CsA/Gef-NPs showed a synergistic effect, rendering 1.6-, 1.9-, and 2.4-fold decreases in the proliferation rates compared with Gef-NPs alone in PC-9, PC-9-GR, and H1975 cells, respectively. Taken together, these four independent assays clearly validate the cytotoxic synergy of a chemosensitizer and an MTA regardless of their co-encapsulation in a nanovehicle.

In vitro mechanism of Gef sensitization by CsA

In accordance with the apoptosis assay, western blot analysis showed that the CsA/Gef-NP treatment increased the level of cPARP (a marker of apoptosis) compared with that induced by Gef-NP treatment, confirming that CsA promoted apoptosis in combination with Gef when co-encapsulated in NPs (Fig. 4g-i). We further explored the mechanism of Gef sensitization by CsA in this nanoplatform. It has been suggested that STAT3 is extensively correlated with therapeutic resistance to EGFR-TKIs in NSCLC cells and that CsA augments the effect of Gef remarkably by inhibiting STAT3 activation. ${ }^{35}$ Therefore, we evaluated whether the CsA-NP-mediated sensitization to Gef is also dependent on STAT3 inhibition in PC-9, PC-9-GR, and H1975 cells by immunoblotting. Consistent with our previous results, the basal levels of STAT3 in drug-resistant PC-9-GR and H1975 cells were much higher than that in Gef-sensitive PC-9 cells (Fig. 4g-i). In PC-9-GR and H1975 cells, Gef-NPs alone had no effect on the p-STAT3 level, while the addition of CsA to the NP formulation resulted in significantly reduced levels of p-STAT3 and its downstream effector Bcl-2. In PC-9 cells, Gef-NP treatment led to the feedback activation of STAT3, which was dramatically suppressed by the addition of CsA to the NP formulation. These results further confirmed that CsA can sensitize cells to Gef when co-delivered by NPs. 
a

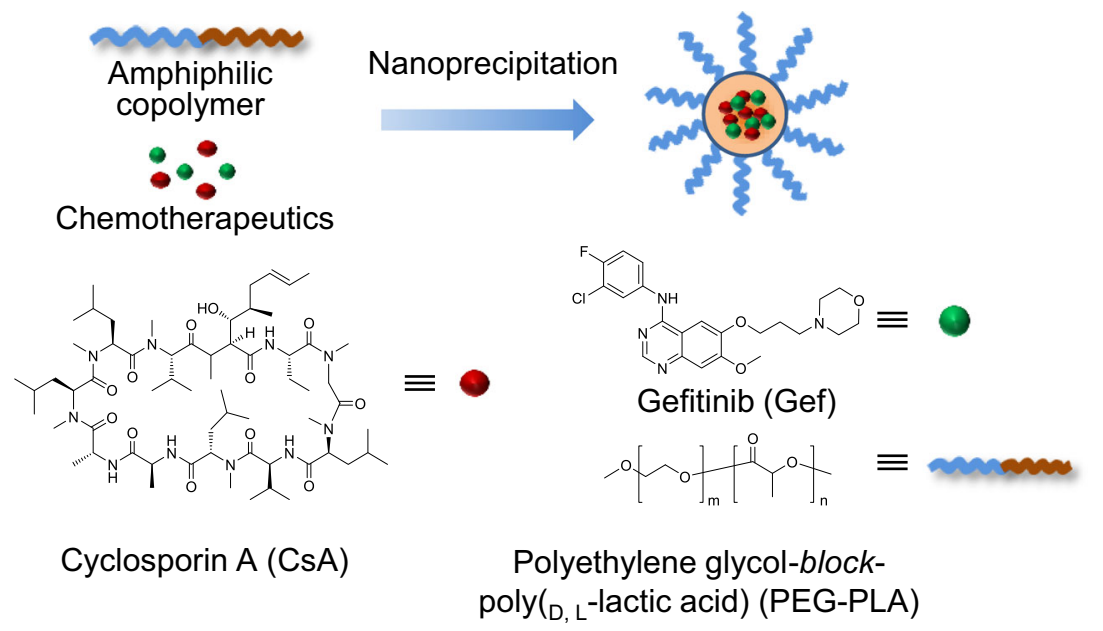

b
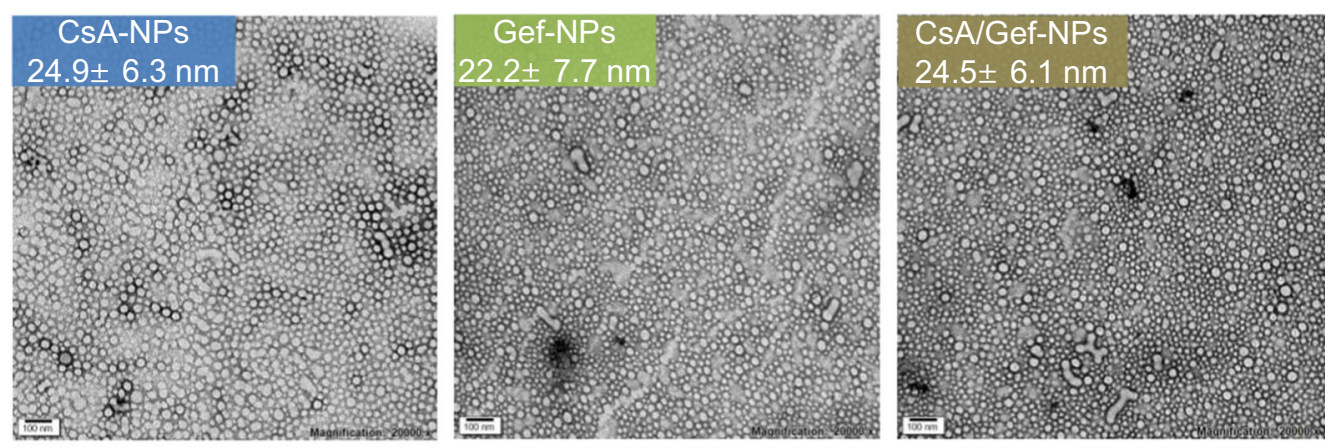

C
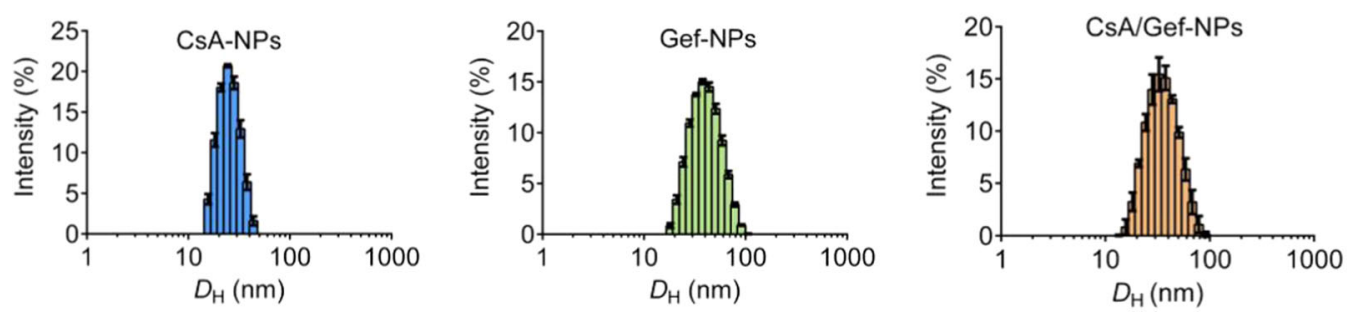

d

\begin{tabular}{|c|c|c|c|}
\hline & DLS $(\mathrm{nm})$ & PDI & Zeta potential $(\mathrm{mv})$ \\
\hline CsA-NPs & $25.7 \pm 6.3$ & $0.270 \pm 0.050$ & $0.619 \pm 0.103$ \\
\hline Gef-NPs & $42.4 \pm 14.9$ & $0.193 \pm 0.013$ & $4.977 \pm 2.459$ \\
\hline CsA/Gef-NPs & $37.1 \pm 13.1$ & $0.106 \pm 0.004$ & $8.160 \pm 9.381$ \\
\hline
\end{tabular}

Fig. 1 a Schematic illustration of the generation of a water-soluble and systemically injectable nanomedicine co-encapsulating two anticancer agents, cyclosporin A (CsA) and Gefitinib (Gef), using the amphiphilic block copolymer polyethylene glycol-block-poly(lactide) (PEG-PLA). The chemical structures of CsA, Gef, and PEG-PLA are also presented. $\mathbf{b}$ Transmission electron microscopy (TEM) morphology of drug-formulated nanoparticles (NPs) (termed CsA-NPs, Gef-NPs, and CsA/Gef-NPs for formulations including CsA, Gef, and both CsA and Gef, respectively). Scale bars represent $100 \mathrm{~nm}$. c Size distribution of NPs as determined by dynamic light scattering (DLS). d DLS analysis indicated that these NPs were small in size and had a narrow size distribution. Zeta potentials are also provided

In vivo antitumor activity overcomes drug resistance in a PC-9-GR tumor-bearing mouse model

To further evaluate the therapeutic efficacy of NPs containing both CsA and Gef, we established an NSCLC xenograft-bearing BALB/c nude mouse model by implanting EGFR-TKI-resistant PC-9$\mathrm{GR}$ cells into immunodeficient mice. When the tumor volume reached approximately $120 \mathrm{~mm}^{3}$, the mice were administered drugs via the tail vein. The therapeutic results are shown in Fig. 5a. Free Gef or Gef-NPs alone did not observably inhibit the growth of
PC-9-GR tumor xenografts that had acquired EGFR-TKI resistance. Similarly, CsA-NPs alone did not effectively suppress tumor growth. As expected, the combination of free CsA and Gef administered by gavage delayed tumor growth compared with free Gef alone. More strikingly, the intravenous (IV)-administered nanomedicine (i.e., CsA/Gef-NPs) suppressed tumor growth more effectively than the conventional regimen of combined oral administration (Fig. 5a). As indicated in Fig. 5c, d, the tumor volume of mice treated with CsA/Gef-NPs was dramatically smaller 
a
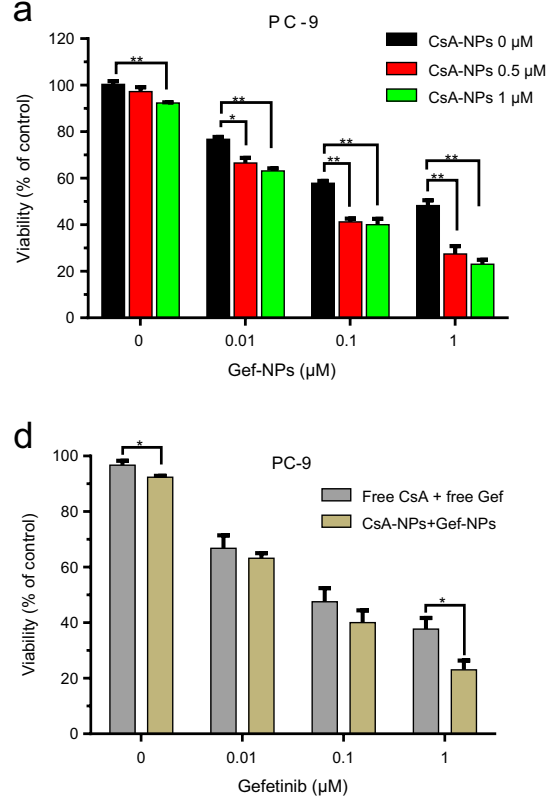

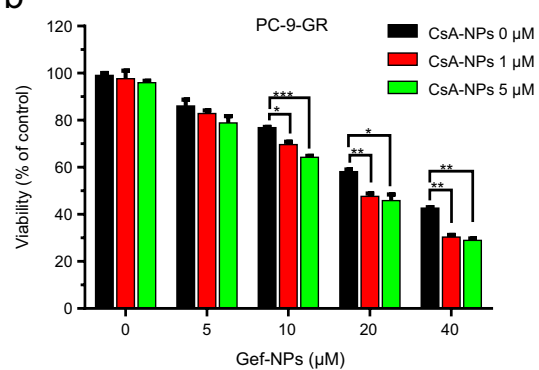

e

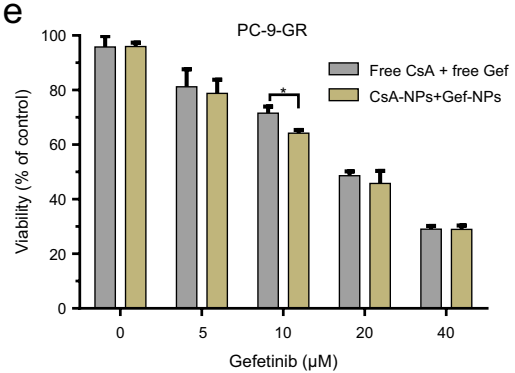

C

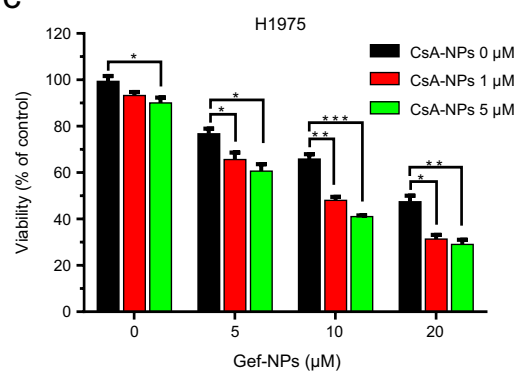

f

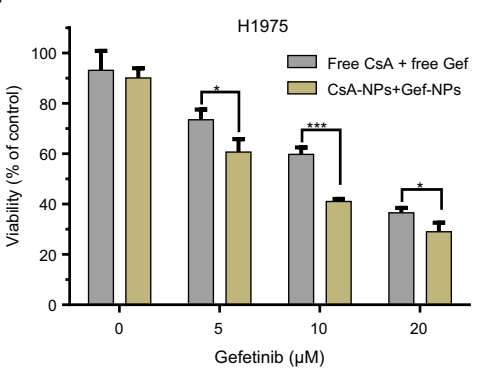

Fig. 2 Cytotoxicity of Gef and CsA and their nanoformulations. The viabilities (mean \pm SD) of PC-9, PC-9-GR, and H1975 lung cancer cells were determined by the MTT assay in the presence or absence of CsA delivered by NPs. (a-c) The cells were treated with various formulations in vitro. (d-f) The in vitro cytotoxicities of the free drug combination and nanodrug combination in successive concentrations were compared in PC-9, PC-9-GR, and H1975 cells. The concentrations of CsA or CsA-NPs included in the nanoformulation or free drugs combination were set to $1 \mu \mathrm{M}, 5 \mu \mathrm{M}$, and $5 \mu \mathrm{M}$ (CsA equivalence) for PC-9, PC-9-GR, and H1975, respectively

than those of mice treated with the combination of free drugs (1.7-fold decrease) and mice treated with saline (2.6-fold decrease) on day 18 post-administration. It should be noted that the total dosage of the CsA plus Gef administered orally was twofold greater than that of the IV injected CsA/Gef-NPs. Furthermore, no significant body weight loss was observed in mice receiving the CsA/Gef-NPs, indicating that the injected NP formulation had low systemic toxicity (Fig. 5b).

In vivo inhibition of STAT3/BCl-2 in mice by CsA-NP treatment Finally, we investigated the effects of CsA-NPs on the STAT3 signaling pathway in nude mice bearing PC-9-GR and H1975 xenograft tumors. The tumor tissues of mice administrated with the drug formulations were collected on day 18 and subjected to histological analysis. In accordance with our previous report $^{35}$ and the in vitro analyses described above, the p-STAT3 levels in mice treated with the combination of free drugs were dramatically lower than those in mice treated with Gef-NPs alone. Moreover, the co-delivery of CsA and Gef in NPs further decreased the p-STAT3 levels compared with the oral administration of the drug combination by 1.52 - and 1.44-fold in H1975 and PC-9-GR xenograft tumors, respectively (Fig. $6 a, b, d, e$ ). To validate the enhanced effect, we analyzed proteins extracted from the excised tumors by western blotting. As expected, the levels of both $p$ STAT3 and downstream Bcl-2 were significantly reduced in mouse tumors after treatment with CsA/Gef-NPs. On the other hand, cPARP was evidently activated in two distinct tumor tissues after treatment with CsA/Gef-NPs, indicating that the inhibition of STAT3 by CsA augmented the Gef-induced apoptosis of tumor cells (Fig. 6c, f), a finding that is consistent with the observed potent suppression of tumor growth.

\section{DISCUSSION}

MDR is one of the largest concerns for cancer therapies. To this end, we have described the co-formulation of the MDR-reversing agent CsA and MTA Gef into a single PEG-PLA NP platform. Characterization of the PEGylated nanoplatform showed that NPs prepared by this method exhibited sub-50-nm diameters (Fig. 1), enabling them to deeply penetrate solid tumors via the EPR effect. Additionally, cloaking the surface with PEG chains not only provides aqueous solubility but also confers the NPs with a "stealth" property, thereby extending the duration of NP circulation in the blood.

The results from a series of in vitro studies demonstrated that the addition of CsA to the NP formulation increased the potency of Gef in non-Gef-resistant cells as well as in primarily and secondarily Gef-resistant cells. The apoptosis, western blotting and EdU assays further showed that the sensitization to Gef by CsA when co-delivered by NPs was a consequence of increased apoptosis and impaired proliferation of NSCLCs. Compared with the free drug combination administered by gavage in vivo, the IVadministered drug combination of CsA and Gef in nanoform significantly decreased tumor growth (Fig. 5c). Furthermore, no significant body weight loss was observed in mice receiving the nanoformulated drug combination, indicating that the injected NP formulation had low systemic toxicity (Fig. 5b).

The mechanisms accounting for the high potency of CsA in sensitizing Gef in the nanoformulation were also investigated. As indicated in Fig. 4g-i, Gef-NPs alone had no effect on the p-STAT3 level in PC-9-GR and H1975 cells, while the addition of CsA to the NP formulation resulted in significantly reduced levels of p-STAT3 and its downstream effector $\mathrm{Bcl}-2$. The same sensitization effect was also witnessed in vivo (Fig. 6). Those mechanisms are summarized in Fig. 7. As a proproliferative, proinvasive and antiapoptotic protein on the cell membrane, the dimerization and autophosphorylation of EGFR stimulate its intrinsic intracellular protein tyrosine kinase activity and initiates downstream pleiotropic oncogenic signaling cascades, including the Ras/MEK/ ERK $^{46,47} \mathrm{PI3K} / \mathrm{Akt} / \mathrm{mTOR}^{25,26,48,49}$ and STAT3 pathways. ${ }^{5,51} \mathrm{Gef}$ inhibits EGFR tyrosine kinase and leads to the disruption of downstream signaling transduction, thus inhibiting the growth and invasion of tumor cells. Exposure to Gef results in the feedback activation of STAT3, which is known to promote tumor cell survival under conditions of stress. However, CsA co-delivered with Gef by NPs is released from the CsA/Gef-NPs and 

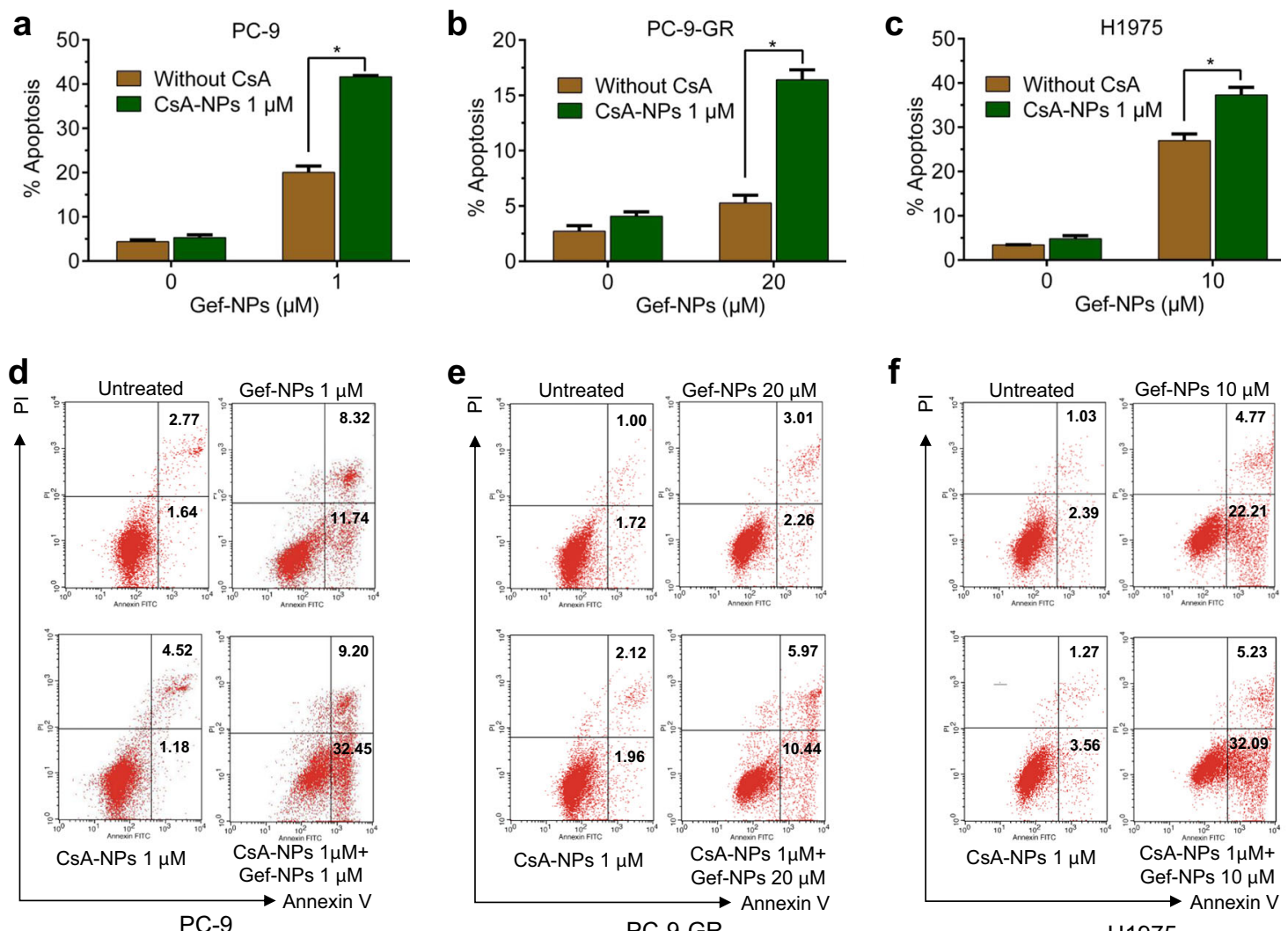

H1975
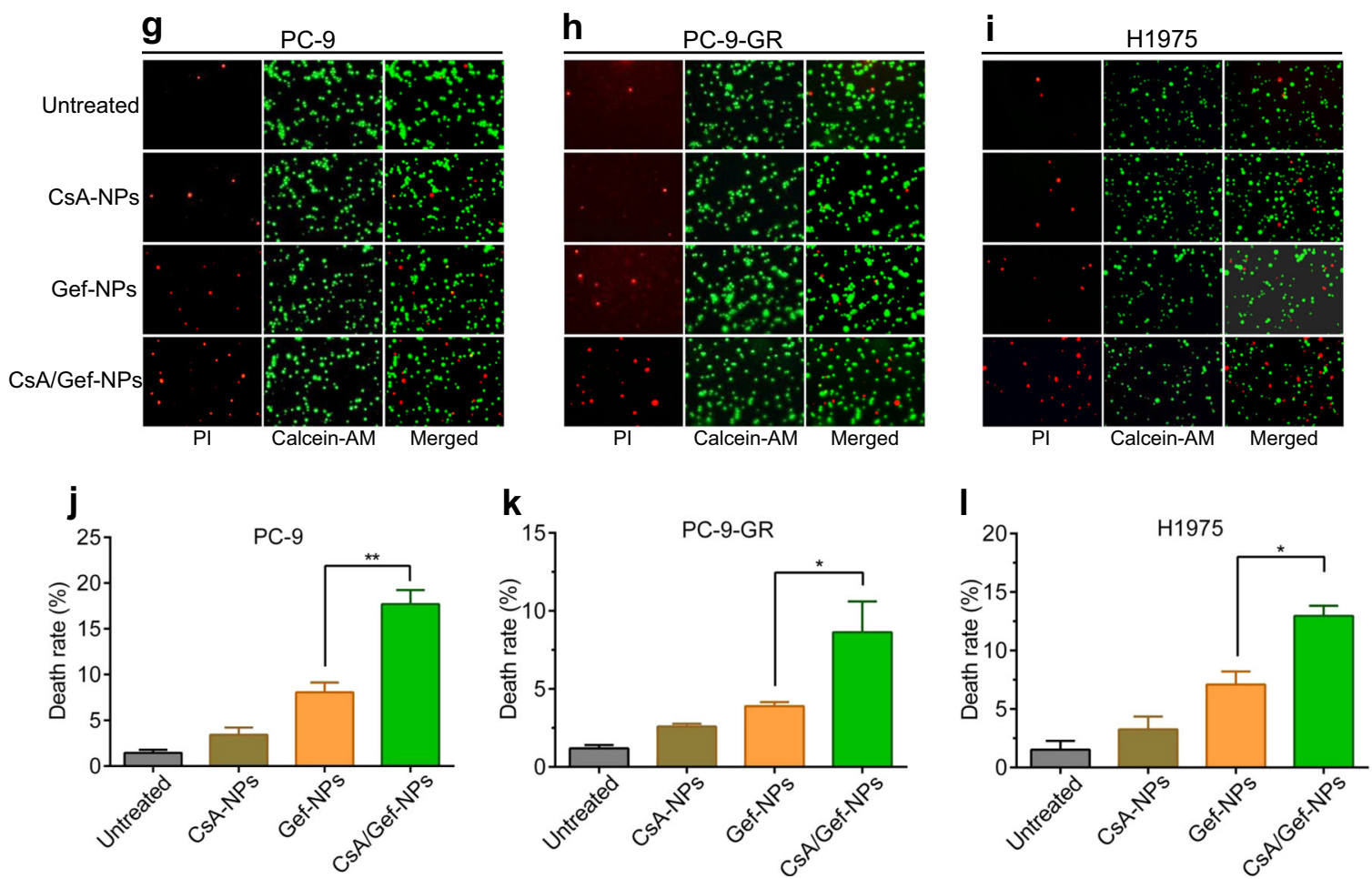

Fig. 3 a-f CSA enhances the cytotoxicity of Gef when co-delivered by NPs to three NSCLC cell lines by promoting apoptosis. Cells were treated with Gef in the absence or presence of CsA via NPs for $48 \mathrm{~h}$ before being stained with Annexin V (AV) and propidium iodide (PI), and the apoptotic rates were determined by flow cytometry. The quantitative apoptotic rates are displayed in the upper panel, and the proportions of apoptotic cells are shown in the lower panel. g-i LIVE/DEAD staining was analyzed by confocal microscopy. PC-9, PC-9-GR, and H1975 NSCLC cells were treated with CsA-NPs $(1,1,1 \mu \mathrm{M})$, Gef-NPs $(1,20,10 \mu \mathrm{M})$, and CsA/Gef-NPs $(1+1,1+20,1+10 \mu \mathrm{M})$. Untreated cells were used as the control. Live cells were stained with calcein-AM, while dead cells were stained with Pl. Fluorescence images of the same samples were captured at $490 \mathrm{~nm}$ (green) for the Calcein-AM signal and at $545 \mathrm{~nm}$ (red) for the PI signal and merged into new images. Scale bars represent $100 \mu \mathrm{m}$. j-I The death rates of PC-9, PC-9-GR and H1975 cells were analyzed. The difference in the death rates of cells treated with CsA-NPs or Gef-NPs alone or in combination was significant 


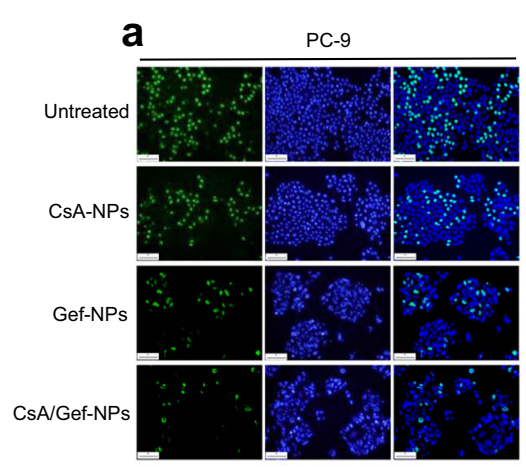

b
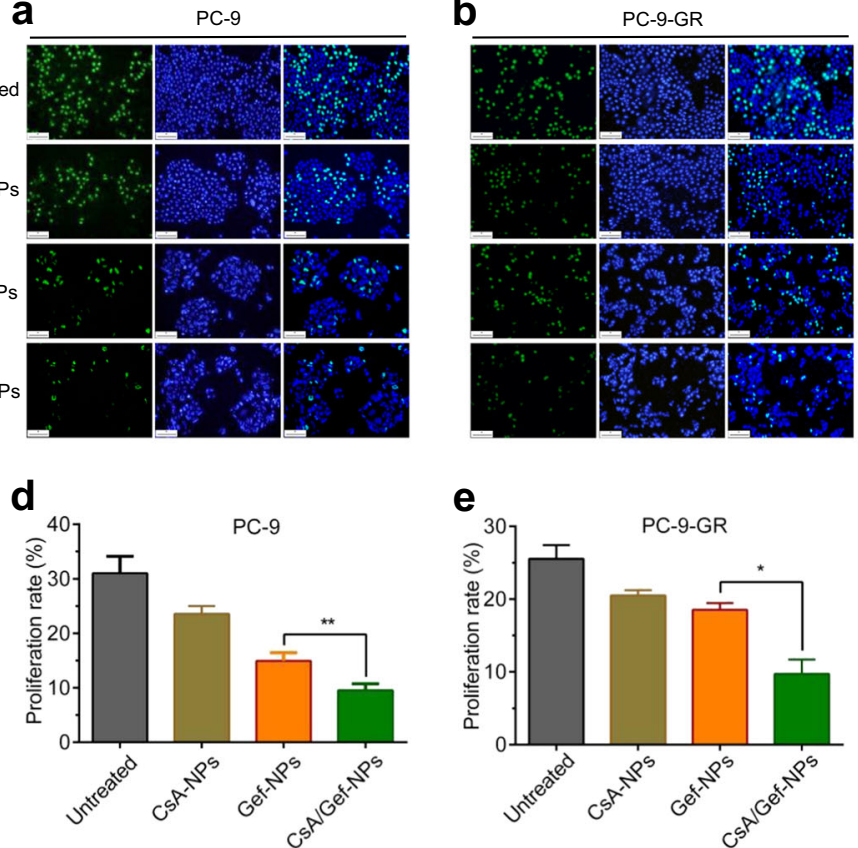

e
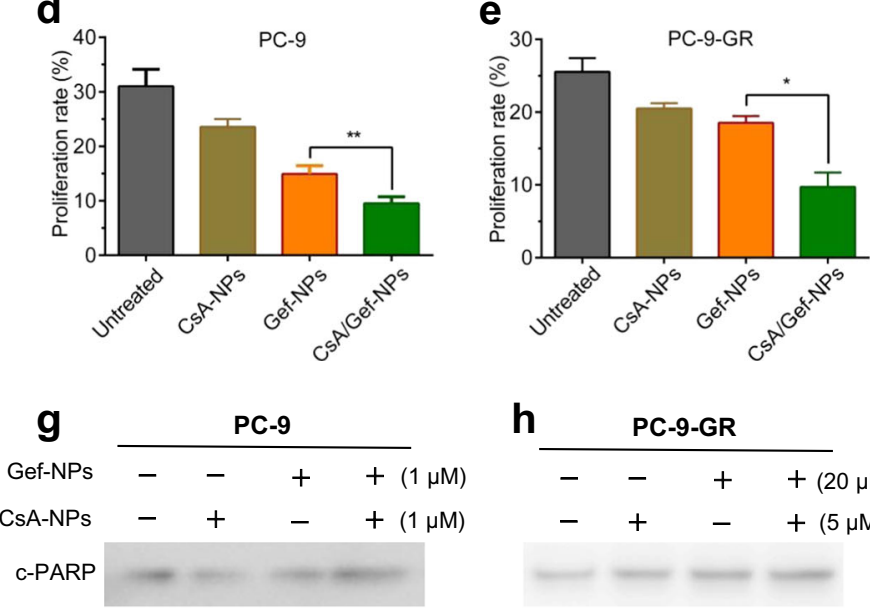

h
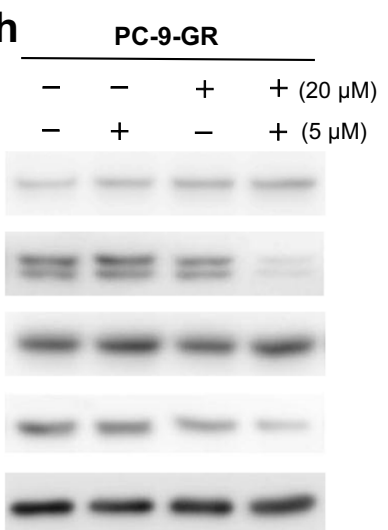

C

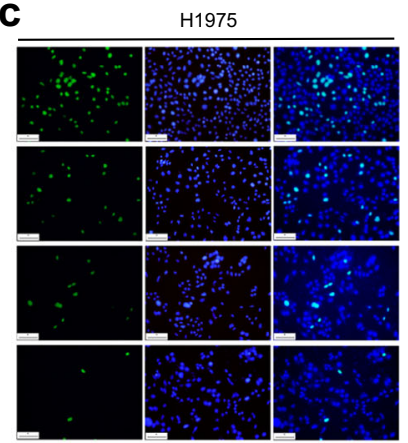

f
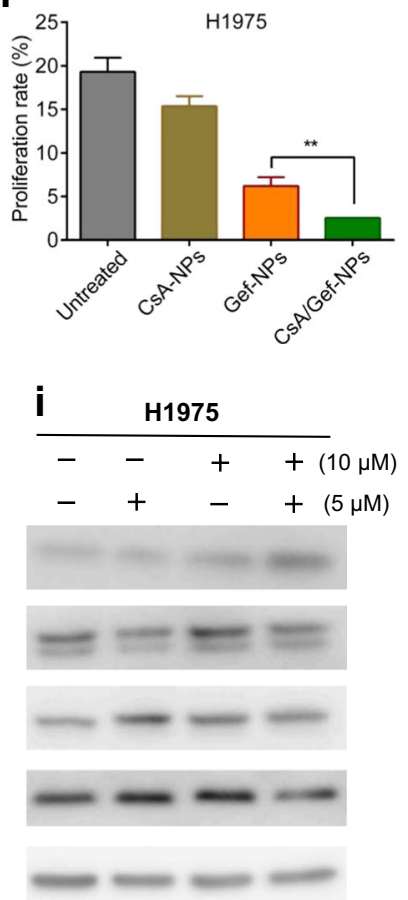

Fig. 4 a-f Inhibitory effect of the nanosystem on cell proliferation. EdU assay results showing the inhibition of cell proliferation by various drugs. a-c The nuclei of all cells were stained with Hoechst 33342 (blue); only proliferating cells were stained by Alexa Fluor 488 (green). The results were analyzed by fluorescence microscopy $(\times 200)$, Scale bars represent $100 \mu \mathrm{m}$. $\mathbf{d}-\mathbf{f}$ The proliferation rates of PC-9, PC-9-GR and H1975 cells treated with CsA-NPs $(1,1,1 \mu \mathrm{M})$, Gef-NPs $(1,20,10 \mu \mathrm{M})$ and CsA/Gef-NPs $(1+1,1+20,1+10 \mu \mathrm{M})$ were quantified and plotted. g-i CsA sensitized NSCLC cells to Gef when co-delivered by NPs via the inhibition of STAT3/Bcl-2. The expression levels of p-STAT3, t-STAT3, and Bcl-2 were examined by western blotting using PC-9, PC-9-GR, and H1975 cell lysates after treatment with Gef-NPs, CsA-NPs, or CsA/Gef-NPs for 48 h. c-PARP, an apoptosis marker, was also detected by immunoblotting

subsequently reverses the activation of STAT3 to effectively augment Gef-induced apoptosis.

In conclusion, our NP-mediated drug codelivery approach successfully overcame MTA resistance by rewiring specific signaling pathways and outperformed the combination of free drugs, enlightening our researchers on overcoming MDR and exploring alternative drug administration routes.

\section{MATERIALS AND METHODS}

Materials

Polyethylene glycol-block-poly (D, L-lactic acid) $\left(\mathrm{PEG}_{5 \mathrm{k}}-b-\mathrm{PLA} \mathrm{A}_{8 \mathrm{k}}\right)$ was purchased from Advanced Polymer Materials, Inc. (Montreal, Canada). Gef (184475-35-2) and CsA (59865-13-3) were purchased from J\&K Scientific (Beijing, China). All other compounds and solvents were purchased from J\&K Chemical (Shanghai, China) and utilized without further purification or dilution.
Preparation and characterization of NPs

Polymeric NPs were prepared via the nanoprecipitation method ${ }^{52-54}$. To fabricate CsA/Gef-NPs, $1 \mathrm{mg}$ of CsA and $40 \mathrm{mg}$ of PEG-PLA were dissolved in $2 \mathrm{ml}$ of acetone, while $1 \mathrm{mg}$ of Gef was dissolved in 0.1 $\mathrm{ml}$ of dimethyl sulfoxide (DMSO). Next, the solution was mixed and added dropwise into $10 \mathrm{ml}$ of DI water while stirring. After stirring for $30 \mathrm{~min}$, the solution was evaporated in a rotary evaporator at reduced pressure at $30^{\circ} \mathrm{C}$ for $\sim 20 \mathrm{~min}$ to remove acetone, yielding a final polymer concentration of $0.1 \mathrm{mg} / \mathrm{mL}$. The transparent solution containing NPs was concentrated with an Amicon Ultra-4 centrifugal filter (MWCO 10 kDa; UFC800324, Millipore, Germany) and washed with DI water. The concentration of copolymer was determined by high-performance liquid chromatography. CsA-NPs and Gef-NPs were prepared by the same method. The $D_{H}$, polydispersity index (PDI), and $\zeta$ potential of the drug-loaded micelles were measured using a Malvern Nano-ZS 90 laser particle size analyzer at $25^{\circ} \mathrm{C}$. The morphological characteristics of the NPs were observed by 

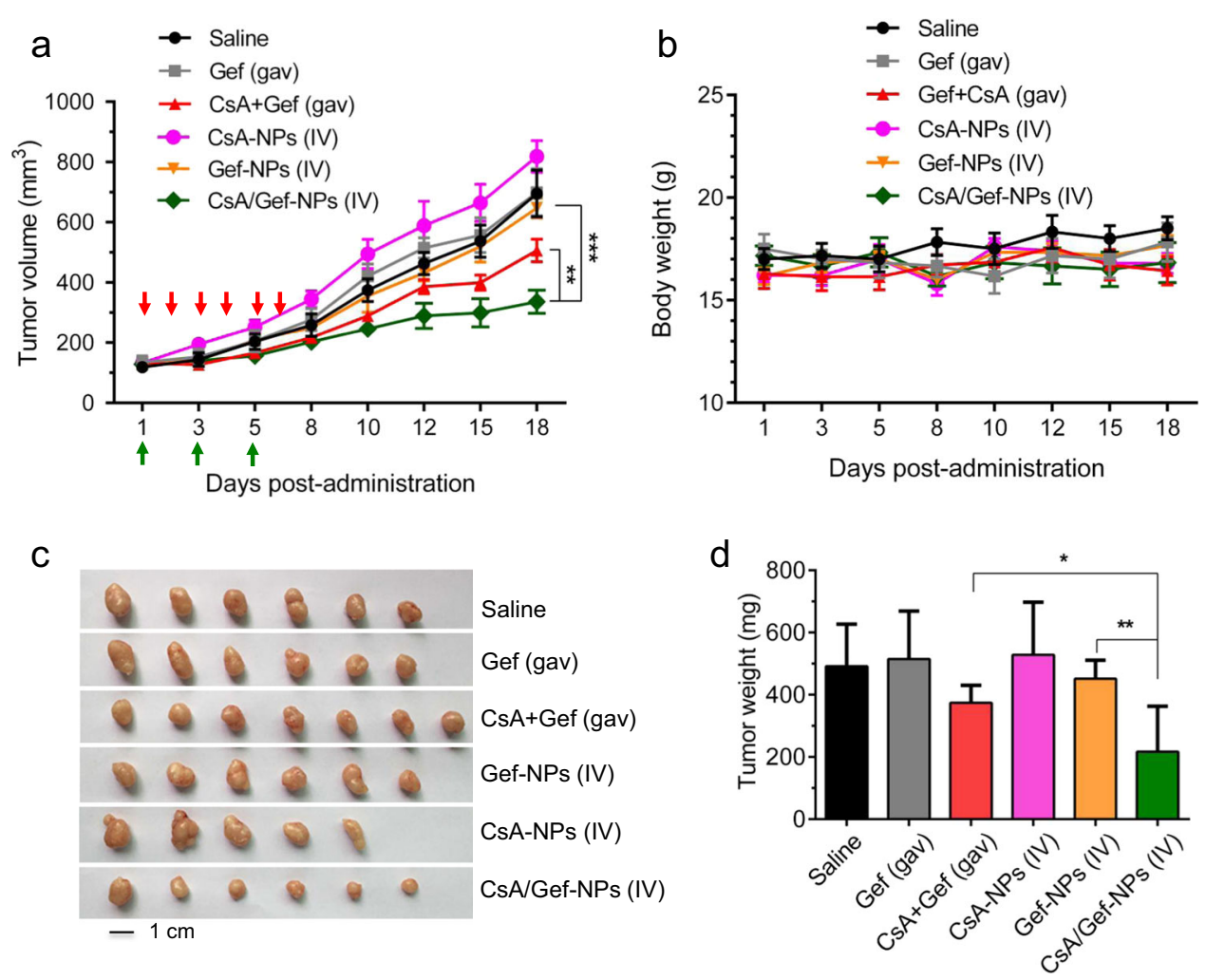

Fig. 5 Co-encapsulation of CsA and Gef results in improved therapeutic efficacy in an acquired EGFR-TKI-resistant PC-9-GR tumor-bearing mouse model. a The tumor volume $\left(\mathrm{mm}^{3}\right)$ change is presented as a function of time after drug treatment. When the tumor volume reached approximately $120 \mathrm{~mm}^{3}$, NP solutions were IV injected three times (indicated by green arrows). Free CsA and/or free Gef were orally administered (shown in red arrows). Saline was intravenously injected as a control. b Body weight gain/loss profiles of mice upon treatment. c Image showing tumors excised from the mice. $\mathbf{d}$ The tumor tissue weight on day 18, the endpoint of the study

transmission electron microscopy (TEM; H-6009IV, Hitachi, Japan). Samples for TEM were stained with $1 \%$ uranyl acetate.

Cell lines and cell culture

The NSCLC cell lines PC-9, which possesses an EGFR-activating mutation exon 19 deletion and H1975, which harbors the EGFRactivating mutation L858R and the resistant mutation T790M, were purchased from the cell bank of the Chinese Academy of Science (Shanghai, China). PC-9-GR cells were obtained according to our previous report. ${ }^{35}$ Cells were maintained in Dulbecco's Modified Eagle's Medium (DMEM; Gibco, Carlsbad, CA, USA) at $37^{\circ}$ $\mathrm{C}$ in a $90 \%$ humidified atmosphere with $5 \% \mathrm{CO}_{2}$. All media were supplemented with $10 \% \mathrm{FBS}$, penicillin (100 units $/ \mathrm{mL}$ ), and streptomycin $(100 \mathrm{mg} / \mathrm{mL})$. Antibiotics, FBS, $0.25 \%(\mathrm{w} / \mathrm{v})$ trypsin, and the $0.03 \%(\mathrm{w} / \mathrm{v})$ EDTA solution were purchased from HyClone.

Cell viability and proliferation assays

MTT assay. Cells were seeded in $96-$ well plates $(5000$ cells and $100 \mu \mathrm{L}$ of media per well) and incubated at $37^{\circ} \mathrm{C}$ overnight. Subsequently, the adherent cells were treated with different concentrations of free Gef, free CsA, CsA-NPs, Gef-NPs, or CsA/GefNPs. Cells treated with medium that contained the same amount of DMSO as the drug treatment group were included as controls. After $48 \mathrm{~h}$ of incubation, $30 \mu \mathrm{L}$ of 3-[4,5-dimethylthiazol-2-yl]-3,5diphenyl tetrazolium bromide (MTT) solution $(5 \mathrm{mg} / \mathrm{mL}$ in PBS) was added to each well. After $4 \mathrm{~h}$ of incubation at $37^{\circ} \mathrm{C}$, the MTT solution was removed from the wells, followed by the addition of $100 \mu \mathrm{L}$ of DMSO per well. The absorbance of individual wells was then measured at $492 \mathrm{~nm}$ using a microplate reader (Multiskan FC, Thermo Scientific) after $2 \mathrm{~h}$ of incubation. All experiments were conducted in triplicate. The $\mathrm{IC}_{50}$ was calculated using GraphPad
Prism 6.0. Untreated cells served as controls, and their viability was defined as $100 \%$. Cell viability was calculated using the following formula: cell viability $=(A$ sample/A control $) \times 100 \%$, where $A$ represents the absorbance at $492 \mathrm{~nm}$. The results were from three repeated independent experiments.

\section{LIVE/DEAD staining assay}

For the LIVE/DEAD staining assay, cells were seeded in 6-well plates at a cell density of $2 \times 10^{5} /$ well. After treatment with different concentrations of NPs for $48 \mathrm{~h}$, the cells in each well were collected and re-suspended in $200 \mu \mathrm{L}$ of PBS. Thereafter, the cells were stained with $1 \mu \mathrm{L}$ of staining solution containing calcein-AM $(2 \mu \mathrm{M})$ and $1 \mu \mathrm{L}$ of PI $(4 \mu \mathrm{M})$, which stains apoptotic/dead cells. After $30 \mathrm{~min}$ of incubation in the dark at $37^{\circ} \mathrm{C}$, the cells were imaged using a fluorescence microscope. The percentage of red (dead) cells among all cells (green + red) represents the cell death ratio.

EdU assay

Cell proliferation and DNA synthesis were determined using a Click-iT ${ }^{\circledR}$ EdU Alexa Fluor ${ }^{\circledR} 488$ Assay Kit (Invitrogen) according to the manufacturer's protocol. Briefly, cells $\left(2 \times 10^{4}\right.$ cells/well) were seeded in 48-well plates and cultured overnight before being exposed to different concentrations of CsA-NPs, Gef-NPs or CsA/ Gef-NPs for $48 \mathrm{~h}$. Cells treated with DMEM alone were used as the control. Next, $100 \mu \mathrm{L}$ of EdU $(10 \mu \mathrm{M})$ was added to each well, followed by $4 \mathrm{~h}$ of incubation at $37^{\circ} \mathrm{C}$. After washing with PBS three times, the cells were fixed with $4 \%$ paraformaldehyde in PBS for $30 \mathrm{~min}$ and permeabilized with $0.5 \%$ Triton X-100 in PBS for 30 min at room temperature. Thereafter, $100 \mu \mathrm{L}$ of Alexa Fluor 488 staining solution was added to each well, followed by 

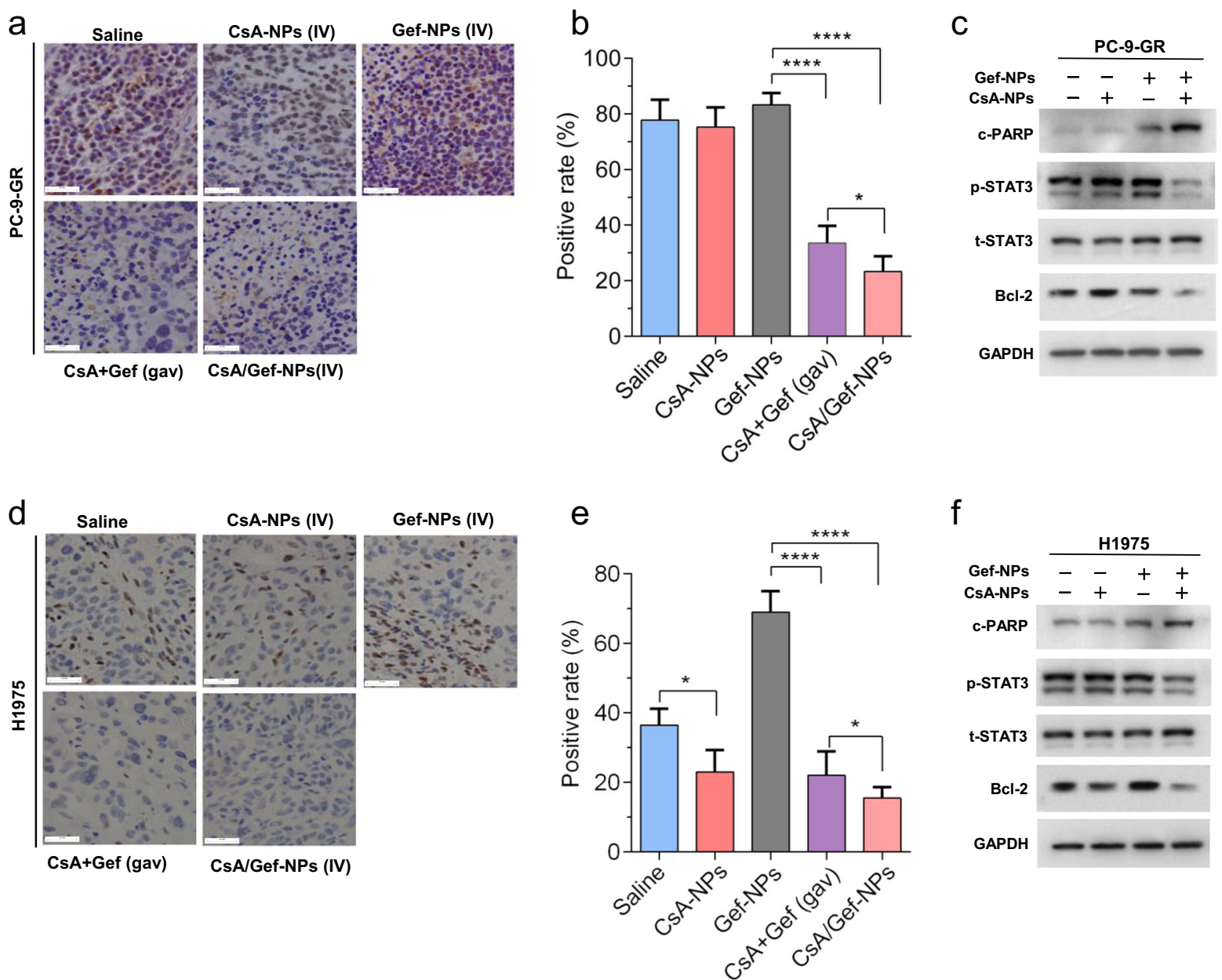

Fig. 6 CsA inhibits Gef-induced STAT3 activation when co-delivered by NPs in two NSCLC xenograft models. Scale bars represent $50 \mu$ m. a-c Immunohistochemical (IHC) staining for p-STAT3 in paraffin-embedded tissue sections, quantitative IHC results, and western blot results for p-STAT3/BCl-2 in PC-9-GR xenograft-bearing mice. $\mathbf{d}-\mathbf{f}$ The same assays were conducted for $\mathrm{H} 1975$ xenografts. The magnification of the IHC images is 400X. c, f Tumor tissue levels of c-PARP, an apoptosis marker, in both PC-9-GR and H1975 xenograft tumors were also detected by immunoblotting

incubation for $30 \mathrm{~min}$ in the dark at room temperature. After the solution was removed, $100 \mu \mathrm{L}$ of Hoechst $33342(5 \mu \mathrm{g} / \mathrm{mL})$ nuclear staining solution was added to each well, followed by incubation for $10 \mathrm{~min}$. Finally, the cells were visualized by fluorescence microscopy. The ratio of EdU-positive cells (green) to all Hoechstpositive cells (blue) represents the proliferation ratio.

Apoptosis analysis using flow cytometry with Annexin V-FITC To analyze apoptosis, cells were seeded in 6-well plates at a density of $2.0 \times 10^{5}$ cells/well and allowed to grow overnight. Next, the cells were treated with different drugs at different concentrations for $48 \mathrm{~h}$ at $37^{\circ} \mathrm{C}$. Untreated cells were used as the control. After incubation, the cells were harvested and washed twice with cold PBS. Next, $1 \times 10^{5}$ cells were dispersed in $100 \mu \mathrm{L}$ of $1 \times$ Annexin V binding buffer. Subsequently, $5 \mu \mathrm{L}$ of Annexin VFITC and $5 \mu \mathrm{L}$ of PI were added, and the cells were incubated at room temperature in the dark for $15 \mathrm{~min}$. Finally, $400 \mu \mathrm{L}$ of $1 \times$ Annexin $\mathrm{V}$ binding buffer was added under gentle mixing, and the samples were analyzed by flow cytometry (BD Biosciences, San Jose, CA).

Western blot analysis

Cells were cultured at a density of $2.0 \times 10^{5}$ cells/well in a 6-well plate and allowed to grow overnight. Next, following treatment with free Gef, free CsA, CsA-NPs, Gef-NPs, or CsA/Gef-NPs, the cells were harvested and lysed in RIPA lysis buffer supplemented with complete protease inhibitor cocktail tablets on ice. Protein was also extracted from the tumor tissues of mice in the in vivo study. Protein concentrations were determined by the bicinchoninic acid protein assay. An equivalent amount of protein was taken from each sample, separated by sodium dodecyl sulfate-polyacrylamide gel electrophoresis, transferred onto polyvinylidene difluoride membranes, and then incubated with antibodies (Cell Signaling Technology) against cleaved poly(ADPribose) polymerase (c-PARP), phosphorylated STAT3 (p-STAT3), total STAT3 (t-STAT3), B cell lymphoma-2 (Bcl-2), and glyceraldehyde-3-phosphate dehydrogenase (GAPDH). The blots were developed with horseradish peroxidase-conjugated secondary anti-rabbit lgG or anti-mouse IgG antibody (Cell Signaling Technology) and visualized with a chemiluminescent substrate on X-ray films (Kodak). Western blotting of each protein was performed at least three times.

In vivo antitumor activity

Four- to five-week-old male BALB/C nude mice were purchased from the Shanghai Experimental Animal Center of the Chinese Academy of Science and used for NSCLC implantation. In total, $100 \mu \mathrm{L}$ of the PC-9-GR and H1975 cell suspensions $\left(5 \times 10^{6}\right.$ cells $)$ 


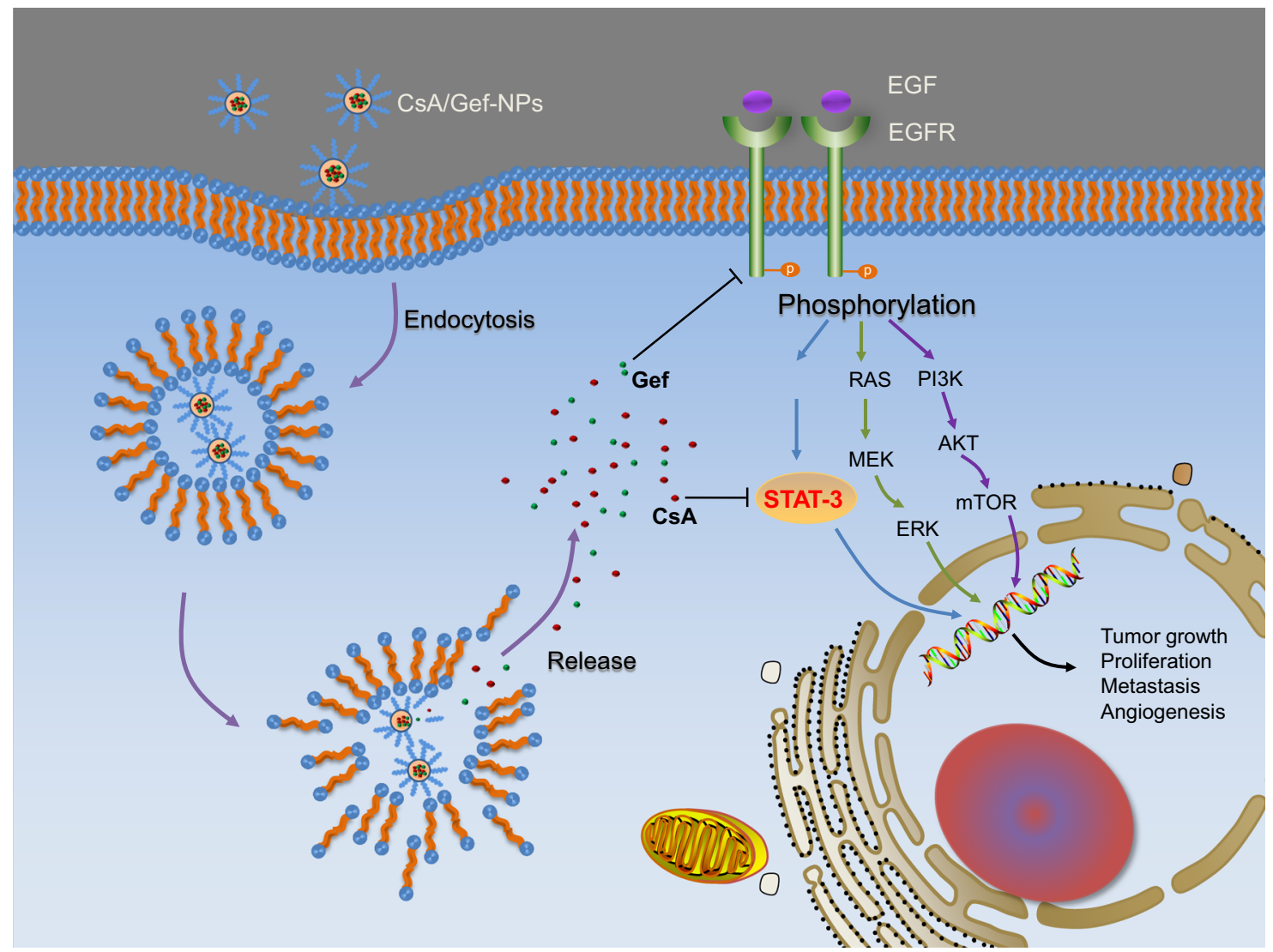

Fig. 7 General mechanism explaining how CsA/Gef-NPs overcome MDR and suppress cancer

was subcutaneously injected into the right flank of each mouse. When the tumor volume reached approximately $120 \mathrm{~mm}^{3}$, the mice were randomized into treatment groups. Mice bearing H1975 xenografts were assigned into five groups for histological study, whereas mice bearing PC-9-GR xenografts were randomized into 11 groups (6 groups for tumor growth evaluation and 5 groups for histological study). Each group contained five to seven mice. NP solutions containing the combination of CsA and Gef (at a dose of $10 \mathrm{mg} / \mathrm{kg}$ for each drug) were IV injected every other day three times, while the combination of the two free drugs was administered by gavage (gav) (at a dose of $20 \mathrm{mg} / \mathrm{kg}$ for each drug) successively for 6 days. CsA-NPs (IV, three times, $10 \mathrm{mg} / \mathrm{kg}$ CsA equivalence), Gef-NPs (IV, three times, $10 \mathrm{mg} / \mathrm{kg}$ Gef equivalence), Gef (gav, six times, $20 \mathrm{mg} / \mathrm{kg}$ ), and saline were included as references. The tumor volume $(V)$ was calculated using the following formula: $V=\left(L \times W^{2}\right) \times 0.5$, where $L$ represents the length, and $W$ represents the width. The weight of each mouse was measured for the evaluation of systemic toxicity. At the end of the study, the mice were sacrificed by $\mathrm{CO}_{2}$ inhalation, and the tumor tissues were fixed in formalin and embedded in paraffin. All the animal protocols were conducted in compliance with the National Institute's Guide for the Care and Use of Laboratory Animals.

Histopathological analysis of tumor tissues Immunohistochemical (IHC) staining using primary antisera and avidin-biotin-peroxidase complex methods were performed using formalin-fixed tumor sections. p-STAT3 expression was monitored using antibodies and visualized by light microscopy. Six fields of view for each sample (magnification, 400x) were randomly selected and analyzed by three pathologists.
Statistical analysis

All the data are presented as means \pm SD and were analyzed using SPSS 17.0 software. The significance of differences was assessed using one-way ANOVA combined with Student's $t$-test $\left({ }^{*} p<0.05\right.$; $\left.{ }^{* *} p<0.01 ;{ }^{* * *} p<0.001\right)$.

\section{ACKNOWLEDGEMENTS}

This work was supported by the National Natural Science Foundation of China (nos 81773193, 81571799, 81572361, and 81372621), the Zhejiang Province Preeminence Youth Fund (no. LR16H160001), and the Zhejiang Medical Innovative Discipline Construction Project-2016.

\section{ADDITIONAL INFORMATION}

The online version of this article (https://doi.org/10.1038/s41392-018-0019-4) contains supplementary material, which is available to authorized users.

Competing interests: The authors declare no competing interests.

\section{REFERENCES}

1. Holohan, C., Van Schaeybroeck, S., Longley, D. B. \& Johnston, P. G. Cancer drug resistance: an evolving paradigm. Nat. Rev. Cancer 13, 714-726 (2013).

2. Zhao, Y. et al. The dual-inhibitory effect of miR-338-5p on the multidrug resistance and cell growth of hepatocellular carcinoma. Signal Transduct. Target Ther. 3, 3 (2018)

3. Wang, N. et al. Paclitaxel and tacrolimus coencapsulated polymeric micelles that enhance the therapeutic effect of drug-resistant ovarian cancer. ACS Appl. Mater. Interfaces 8, 4368-4377 (2016).

4. Xing, Y., Zhang, J., Chen, F., Liu, J. \& Cai, K. Mesoporous polydopamine nanoparticles with co-delivery function for overcoming multidrug resistance via synergistic chemo-photothermal therapy. Nanoscale 9, 8781-8790 (2017). 
5. Robey, R. W. et al. Revisiting the role of $A B C$ transporters in multidrug-resistant cancer. Nat. Rev. Cancer. https://doi.org/10.1038/s41568-018-0005-8. (2018).

6. Wang, $H$. et al. Biocompatible, chimeric peptide-condensed supramolecular nanoparticles for tumor cell-specific siRNA delivery and gene silencing. Chem. Commun. 50, 7806-7809 (2014).

7. Chen, Z., Krishnamachary, B., Penet, M. F. \& Bhujwalla, Z. M. Acid-degradable dextran as an image guided siRNA carrier for COX-2 downregulation. Theranostics 8, 1-12 (2018).

8. Ahn, H. J. Abstract 2139: RNA nanoparticles as a two-in-one siRNA delivery carrier synergistically suppress drug-resistance cancer via combination therapy with doxorubicin. Cancer Res. 76, 2139-2139 (2016).

9. Roberts, C. M. et al. Nanoparticle delivery of siRNA against TWIST to reduce drug resistance and tumor growth in ovarian cancer models. Nanomedicine 13, 965-976 (2016).

10. Shi, L., Pan, H., Liu, Z., Xie, J. \& Han, W. Roles of PFKFB3 in cancer. Signal Transduct. Target Ther. 2, 17044 (2017).

11. Yan, Y., Björnmalm, M. \& Caruso, F. Particle carriers for combating multidrugresistant cancer. ACS Nano 7, 9512-9517 (2013).

12. Wang, B. et al. Cationic oligo(p-phenylene vinylene) materials for combating drug resistance of cancer cells by light manipulation. Adv. Mater. 26, 5986-5990 (2014).

13. Gupta, B. et al. Development of bioactive PEGylated nanostructured platforms for sequential delivery of doxorubicin and imatinib to overcome drug resistance in metastatic tumors. ACS Appl. Mater. Interfaces 9, 9280-9290 (2017).

14. Chen, Y., Chen, H. \& Shi, J. Inorganic nanoparticle-based drug codelivery nanosystems to overcome the multidrug resistance of cancer cells. Mol. Pharm. 11, 2495-2510 (2014)

15. Kong, F. et al. Gold nanorods, DNA origami, and porous silicon nanoparticlefunctionalized biocompatible double emulsion for versatile targeted therapeutics and antibody combination therapy. Adv. Mater. 28, 10195-10203 (2016).

16. Yang, R., Mondal, G., Wen, D. \& Mahato, R. I. Combination therapy of paclitaxel and cyclopamine polymer-drug conjugates to treat advanced prostate cancer. Nanomedicine 13, 391-401 (2016).

17. Wang, $\mathrm{H}$. et al. New generation nanomedicines constructed from self-assembling small molecule prodrugs alleviate cancer drug toxicity. Cancer Res. 24, 6963-6974 (2017).

18. Yang, R. et al. Getting drugs across biological barriers. Adv. Mater. 37, 1606596 (2017).

19. Hansen, A. E. et al. Positron emission tomography based elucidation of the enhanced permeability and retention effect in dogs with cancer using copper-64 liposomes. ACS Nano 9, 6985-6995 (2015).

20. Nel, A., Ruoslahti, E. \& Meng, H. New insights into "permeability" as in the enhanced permeability and retention effect of cancer nanotherapeutics. ACS Nano 11, 9567-9569 (2017).

21. Santini, C. et al. Intravenous and intratumoral injection of Pluronic P94: the effect of administration route on biodistribution and tumor retention. Nanomedicine 13, 2179-2188 (2017)

22. Senapati, S., Mahanta, A. K., Kumar, S. \& Maiti, P. Controlled drug delivery vehicles for cancer treatment and their performance. Signal Transduct. Target Ther. 3, 7 (2018).

23. Nicolas-Boluda, A., Silva, A. K. A., Fournel, S. \& Gazeau, F. Physical oncology: new targets for nanomedicine. Biomaterials 150, 87-99 (2017).

24. Au, K. M. et al. Improving cancer chemoradiotherapy treatment by dual controlled release of wortmannin and docetaxel in polymeric nanoparticles. ACS Nano 9, 8976-8996 (2015).

25. Kwon, K. C. et al. Superparamagnetic gold nanoparticles synthesized on protein particle scaffolds for cancer theragnosis. Adv. Mater. 38, 1701146 (2017).

26. Wang, $\mathrm{H}$. et al. Structure-based rational design of prodrugs to enable their combination with polymeric nanoparticle delivery platforms for enhanced antitumor efficacy. Angew. Chem. Int. Ed. Engl. 53, 11532-11537 (2014).

27. Wang, H. X. et al. Cancer nanomedicines stabilized by pi-pi stacking between heterodimeric prodrugs enable exceptionally high drug loading capacity and safer delivery of drug combinations. Theranostics 7, 3638-3652 (2017).

28. Wang, J. et al. iRGD-decorated polymeric nanoparticles for the efficient delivery of vandetanib to hepatocellular carcinoma: preparation and in vitro and in vivo evaluation. ACS Appl. Mater. Interfaces 8, 19228-19237 (2016).

29. Yang, S. et al. Amphiphilic copolymer coated upconversion nanoparticles for near-infrared light-triggered dual anticancer treatment. Nanoscale 6, 14903-14910 (2014).

30. Yao, Y. J. et al. Perfluorocarbon-encapsulated PLGA-PEG emulsions as enhancement agents for highly efficient reoxygenation to cell and organism. ACS Appl. Mater. Interfaces 7, 18369-18378 (2015).

31. Cui, Y. et al. Dual-targeting magnetic PLGA nanoparticles for codelivery of paclitaxel and curcumin for brain tumor therapy. ACS Appl. Mater. Interfaces 8 , 32159-32169 (2016)
32. Yu, H., Pardoll, D. \& Jove, R. STATs in cancer inflammation and immunity: a leading role for STAT3. Nat. Rev. Cancer 9, 798-809 (2009).

33. Li Li. et al. Epigenomic characterization of a p53-regulated 3p22.2 tumor suppressor that inhibits STAT3 phosphorylation via protein docking and is frequently methylated in esophageal and other rcinomas. Theranostics 8, 61-77 (2018).

34. Doshi, U. A. et al. STAT3 mediates C6-ceramide-induced cell death in chronic lymphocytic leukemia. Signal Transduct. Target Ther. 2, 17051 (2017).

35. Shou, J. et al. Cyclosporine A sensitizes human non-small cell lung cancer cells to gefitinib through inhibition of STAT3. Cancer Lett. 379, 124-133 (2016).

36. Survase, S. A., Kagliwal, L. D., Annapure, U. S. \& Singhal, R. S. Cyclosporin A - a review on fermentative production, downstream processing and pharmacological applications. Biotechnol. Adv. 29, 418-435 (2011).

37. Monegal, A. et al. Bone mass and mineral metabolism in liver transplant patients treated with FK506 or cyclosporine A. Calcif. Tissue Int. 68, 83-86 (2001).

38. Nicolas, J., Mura, S., Brambilla, D., Mackiewicz, N. \& Couvreur, P. Design, functionalization strategies and biomedical applications of targeted biodegradable/ biocompatible polymer-based nanocarriers for drug delivery. Chem. Soc. Rev. 42 , 1147-1235 (2013)

39. Rabanel, J. M. et al. Effect of the polymer architecture on the structural and biophysical properties of PEG-PLA nanoparticles. ACS Appl. Mater. Interfaces 7, 10374-10385 (2015).

40. Coumes, F. et al. Design and development of immunomodulatory antigen delivery systems based on peptide/PEG-PLA conjugate for tuning immunity. Biomacromolecules 16, 3666-3673 (2015).

41. Fahr, D. A. Cyclosporin clinical pharmacokinetics. Clin. Pharmacokinet. 24, 472-495 (1993)

42. Bergman, E. et al. Pharmacokinetics of gefitinib in humans: the influence of gastrointestinal factors. Inter J. Pharm. 341, 134-142 (2007).

43. Qadir, M. et al. Cyclosporin $A$ is a broad-spectrum multidrug. resistance modulator. Clin. Cancer Res. 11, 2320-2326 (2005).

44. Loor, F., Tiberghien, F. O., Wenandy, T., Didier, A. \& Traber, R. Cyclosporins: structure-activity relationships for the inhibition of the human MDR1 Pglycoprotein ABC transporter. J. Med. Chem. 45, 4598-4612 (2002).

45. Spreafico, A. et al. Rational combination of a MEK inhibitor, selumetinib, and the Wnt/Calcium pathway modulator, cyclosporin A, in preclinical models of colorectal cancer. Clin. Cancer Res. 19, 4149-4162 (2013).

46. Sangrar, W. et al. Amplified Ras-MAPK signal states correlate with accelerated EGFR internalization, cytostasis and delayed HER2 tumor onset in Fer-deficient model systems. Oncogene 34, 4109-4117 (2015).

47. Van Emburgh, B. O. et al. Acquired RAS or EGFR mutations and duration of response to EGFR blockade in colorectal cancer. Nat. Commun. 7, 13665 (2016).

48. Liu, X. L. et al. ING5 knockdown enhances migration and invasion of lung cancer cells by inducing EMT via EGFR/PI3K/Akt and IL-6/STAT3 signaling pathways. Oncotarget 8, 54265-54276 (2017).

49. Fan, Q. W. et al. EGFR signals to mTOR through PKC and independently of Akt in glioma. Sci. Signal. 2, ra4 (2009).

50. Li, R. et al. Secreted GRP78 activates EGFR-SRC-STAT3 signaling and confers the resistance to sorafeinib in HCC cells. Oncotarget 8, 19354-19364 (2017).

51. Wu, J., Liu, W., Williams, J. P. \& Ratner, N. EGFR-Stat3 signalling in nerve glial cells modifies neurofibroma initiation. Oncogene 36, 1669-1677 (2017).

52. Miao, Q. et al. Molecular afterglow imaging with bright, biodegradable polymer nanoparticles. Nat. Biotechnol. 35, 1102-1110 (2017).

53. Wu, J. et al. Hyaluronic acid coated PLGA nanoparticulate docetaxel effectively targets and suppresses orthotopic human lung cancer. J. Control Release 259, 76-82 (2017).

54. Saad, W. S. \& Prud'Homme, R. K. Principles of nanoparticle formation by Flash Nanoprecipitation. Nano Today 11, 212-227 (2016).

Open Access This article is licensed under a Creative Commons Attribution 4.0 International License, which permits use, sharing, adaptation, distribution and reproduction in any medium or format, as long as you give appropriate credit to the original author(s) and the source, provide a link to the Creative Commons license, and indicate if changes were made. The images or other third party material in this article are included in the article's Creative Commons license, unless indicated otherwise in a credit line to the material. If material is not included in the article's Creative Commons license and your intended use is not permitted by statutory regulation or exceeds the permitted use, you will need to obtain permission directly from the copyright holder. To view a copy of this license, visit http://creativecommons. org/licenses/by/4.0/.

(c) The Author(s) 2018 